\title{
LARGE DEVIATIONS PROBLEM FOR RANDOM EVOLUTION PROCESSES
}

UDC 519.21

\author{
V. S. KOROLIUK AND I. V. SAMOILENKO
}

\begin{abstract}
This is a short survey of the joint author results concerning the large deviations problem for some stochastic processes of random evolution published in the papers [5]-[20].
\end{abstract}

\section{INTRODUCTION}

This is a short survey of the joint author results concerning the large deviations problem for some stochastic processes of random evolution published in the papers [5]20].

The large deviations problem is often applied to estimate exponentially small probabilities and is a part of the theory of limit theorems for stochastic processes.

We distinguish between the following four types of limit theorems.

I: Averaging or law of large numbers.

II: Diffusion approximation or central limit theorem.

III: Poisson approximation (for semimartingales).

IV: Large deviations or asymptotically small probabilities.

Let $L$ be the generator of a Markov process $\eta(t), t \geq 0$, defined on a standard phase space $(E, \mathcal{E})$, that is, $E$ is a Polish space and $\mathcal{E}$ is its Borel $\sigma$-algebra. Assume that the domain $\mathcal{D}(L)$ of the generator $L$ is dense in $\mathcal{B}_{E}$ and contains functions with continuous derivatives. Here, $\mathcal{B}_{E}$ denotes the Banach space of real-valued finite test functions

$$
\varphi(u) \in E
$$

equipped with the norm

$$
\|\varphi\|:=\sup _{u \in E}|\varphi(u)| .
$$

Limit theorems for Markov processes are based on the martingale characterization of the process $\eta(t), t \geq 0$ :

$$
\mu(t):=\varphi(\eta(t))-\varphi(\eta(0))-\int_{0}^{t} L \varphi(\eta(s)) d s,
$$

2010 Mathematics Subject Classification. Primary 60J55, 60B10, 60F17, 60K10; Secondary 60G46, $60 \mathrm{G} 60$.

Key words and phrases. Large deviations, random evolution processes, asymptotically small diffusion, Lévy approximation, exponential nonlinear generator, split and double merging.

This paper was prepared following the talk at the International conference "Probability, Reliability and Stochastic Optimization (PRESTO-2015)" held in Kyiv, Ukraine, April 7-10, 2015. 
where $\mu(t)$ is a martingale, and

$$
\begin{gathered}
L \varphi(u):=\lim _{\Delta \rightarrow 0} \frac{1}{\Delta} \mathrm{E}[\varphi(u+\Delta \eta(t))-\varphi(u) \mid \eta(t)=u], \\
\Delta \eta(t):=\eta(t+\Delta)-\eta(t)
\end{gathered}
$$

(more detail is given, for example, in [1]).

Similarly, the large deviations problem for Markov processes is based on the exponential martingale characterization of the process $\eta(t), t \geq 0$ :

$$
\mu_{e}(t):=\exp \left\{\varphi(\eta(t))-\varphi(\eta(0))-\int_{0}^{t} H \varphi(\eta(s)) d s\right\},
$$

where $\mu_{e}(t)$ is a martingale, and

$$
H \varphi(u):=e^{-\varphi(u)} L e^{\varphi(u)}, \quad e^{\varphi(x)} \in \mathcal{D}(L),
$$

(see [2] and [3, Chapter 1]).

\section{LARGe DeViations PROBlem For Markov PROCESSES}

The large deviations problem for Markov processes can be solved by using a limit theorem for the exponential generator (2) (the reader may consult the monograph [3] concerning appropriate sufficient conditions and methods used in the framework of this approach). The latter limit theorem states that there exists an operator $H$ such that

$$
H^{\varepsilon} \varphi(u):=e^{-\varphi(u) / \varepsilon} \varepsilon L^{\varepsilon} e^{\varphi(u) / \varepsilon} \rightarrow H \varphi(u), \quad \varepsilon \rightarrow 0 .
$$

Limit theorem (3) is related to the asymptotic behavior of the nonlinear semigroup as $\varepsilon \rightarrow 0$ :

$$
H_{t}^{\varepsilon} \varphi(u):=\varepsilon \ln \mathrm{E}\left[e^{\varphi\left(\eta^{\varepsilon}(t)\right) / \varepsilon} \mid \eta^{\varepsilon}(0)=u\right] .
$$

Remark 2.1. The relationship between the limit theorem (3) for the exponential generator and large deviations problem is not trivial. Namely, the method of solution of the large deviations problem for a normalized Markov process $\eta_{\varepsilon}(t), t \geq 0$, for the convergence as $\varepsilon \rightarrow 0+$ is reduced to verifying the large deviations principle. The latter principle holds if there exists a lower semicontinuous function $I: E \rightarrow[0, \infty)$ such that

$$
\liminf _{\varepsilon \rightarrow 0} \varepsilon \ln \mathrm{P}\left\{\eta_{\varepsilon}(t) \in A\right\} \geq-\inf _{u \in A} I(u)
$$

for an arbitrary open set $A$, and

$$
\limsup _{\varepsilon \rightarrow 0} \varepsilon \ln \mathrm{P}\left\{\eta_{\varepsilon}(t) \in B\right\} \leq-\inf _{u \in B} I(u)
$$

for an arbitrary closed set $B$. Then $I$ is called the rate functional for the large deviations principle.

The solution of the large deviations problem for Markov processes presented in the monograph [3, Chapter 2] consists of the following four steps:

Step 1. Verify the convergence of the exponential (nonlinear) generator $\mathbf{H}^{\varepsilon}$ to the limit exponential (nonlinear) generator $\mathbf{H}$.

Step 2. Verify the exponential compactness of the prelimit Markov process.

Note that the convergence of the semigroups corresponding to $\mathbf{H}^{\varepsilon}$ together with the exponential compactness of the prelimit Markov process implies the large deviations principle in the space $\mathbf{D}_{E}[0, \infty)$.

Step 3. Verify the comparison principle for the limit exponential generator which implies that the semigroups related to the prelimit generator $\mathbf{H}^{\varepsilon}$ converge to a unique semigroup corresponding to the limit generator $\mathbf{H}$. 
Step 4. Construct the variational representation for the limit exponential generator which yields an explicit expression for the rate functional.

Steps 2-4 are demonstrated in the monograph [3] under rather general conditions imposed on the exponential generators corresponding to stochastic processes with independent increments; the latter class of processes is studied in detail in the papers [5][20]. Namely, the exponential compactness of stochastic processes with independent increments is considered in Examples 1.5 and 4.23 and in Sections 10.1.2 and 10.3.2 of [3], while the comparison principle for the corresponding limit exponential generator is considered in Section 10.1.3 by using Lemma 9.15 (see [3]). Finally, the variational representation for the limit exponential generator is obtained in Sections 10.1.5 by using Theorem 8.14 [3]. The methods used in the monograph [3] and briefly mentioned above are applied for both the Lévy approximation and the scheme of small diffusion described below.

Therefore, our aim is to check the convergence of the exponential (nonlinear) generator as described in Step 1 above.

Remark 2.2. Some other approaches to the problems above can be found in the classical monograph 4, where the large deviations problem is solved by using the cumulant of a stochastic process with independent increments.

\section{Limit THEOREMS FOR THE EXPONENTIAL GENERATOR (3)}

We start with the simplest example of Markov processes.

\subsection{Asymptotically small diffusion. Let}

$$
\eta^{\varepsilon}(t)=\sqrt{\varepsilon} \sigma w(t)
$$

where $w(t)$ is a Wiener process. Here,

$$
\begin{gathered}
L^{\varepsilon} \varphi(u)=\varepsilon \frac{\sigma^{2}}{2} \varphi^{\prime \prime}(u), \quad H^{\varepsilon} \varphi(u)=H \varphi(u)+R_{\varepsilon} \varphi(u), \quad R_{\varepsilon} \varphi(u) \rightarrow 0, \quad \varepsilon \rightarrow 0, \\
H \varphi(u)=\frac{\sigma^{2}}{2}\left[\varphi^{\prime}(u)\right]^{2} .
\end{gathered}
$$

3.2. Stochastic processes with independent increments. Let

$$
L^{\varepsilon} \varphi(u)=\varepsilon^{-3} \int_{\mathbf{R}}\left[\varphi\left(u+\varepsilon^{2} v\right)-\varphi(u)\right] L(d v)
$$

and assume the balance condition $\int_{\mathbf{R}} v L(d v)=0$. Here,

$$
\begin{gathered}
H^{\varepsilon} \varphi(u)=H \varphi(u)+R_{\varepsilon} \varphi(u), \quad R_{\varepsilon} \varphi(u) \rightarrow 0, \quad \varepsilon \rightarrow 0, \\
H \varphi(u)=\frac{\sigma^{2}}{2}\left[\varphi^{\prime}(u)\right]^{2}, \quad \sigma^{2}:=\int_{\mathbf{R}} v^{2} L(d v) .
\end{gathered}
$$

3.3. The scheme of the Poisson approximation [13]- 20]. Let

$$
\begin{gathered}
\Gamma_{\delta}^{\varepsilon} \varphi(u)=\varepsilon^{-2} \int_{\mathbf{R}}[\varphi(u+\varepsilon v)-\varphi(u)] \Gamma_{\delta}(d v), \\
\Gamma_{\delta}^{\varepsilon} \varphi(u)=\Gamma \varphi(u)+R_{\varepsilon, \delta} \varphi(u), \quad R_{\varepsilon, \delta} \rightarrow 0, \quad \varepsilon, \delta \rightarrow 0, \quad \varepsilon^{-1} \delta \rightarrow 1, \\
\Gamma \varphi(u)=b \varphi^{\prime}(u)+\int_{\mathbf{R}}\left[\varphi(u+v)-\varphi(u)-v \varphi^{\prime}(u)\right] \Gamma_{0}(d v),
\end{gathered}
$$

where the measure $\Gamma_{0}(\cdot)$ is constructed from the measure $\Gamma_{\delta}(\cdot)$ as follows:

$$
\Gamma_{g}^{\delta}=\int_{\mathbf{R}} g(v) \Gamma_{\delta}(d v)=\delta\left[\Gamma_{g}+\theta_{g}^{\varepsilon}\right], \quad \Gamma_{g}=\int_{\mathbf{R}} g(v) \Gamma_{0}(d v) .
$$


Then

$$
\begin{gathered}
H^{\varepsilon, \delta} \varphi(u)=H \varphi(u)+\theta^{\varepsilon, \delta} \varphi(u), \quad \theta^{\varepsilon, \delta} \rightarrow 0, \quad \varepsilon, \delta \rightarrow 0, \quad \varepsilon^{-1} \delta \rightarrow 1 \\
H \varphi(u)=b \varphi^{\prime}(u)+\int_{\mathbf{R}}\left[e^{v \varphi^{\prime}(u)}-1\right] \Gamma_{0}(d v) .
\end{gathered}
$$

\section{The LARGe DeViations PROBlem FOR RANDOM EVOLUtion PROCESSES}

In what follows we study random evolutions in the Euclidean space, that is, $\eta(t ; x) \in \mathbf{R}$ (the generalization to the case of $\mathbf{R}^{d}$ is rather trivial) driven by a stationary Markov process in a general Banach space, $æ(t) \in E$.

4.1. Random evolution process in a Markov random environment. The environment is described by a stationary Markov process $æ(t), t \geq 0$, defined on a measurable phase space $(E, \mathcal{E})$. The process itself is described by its generator

$$
Q \varphi(\cdot, x)=q \int_{E}[\varphi(y)-\varphi(x)] P(x, d y), \quad x \in E .
$$

The two component Markov process $\eta(t ; x), æ(t), t \geq 0$, is described by the generator

$$
L \varphi(u, x)=[Q+\Gamma(x)] \varphi(u, x)
$$

where $\Gamma(x), x \in E$, defines the associated Markov process $\eta(t), t \geq 0$ :

$$
\Gamma(x) \varphi(u, \cdot)=\int_{\mathbf{R}}[\varphi(u+v)-\varphi(u)] \Gamma(d v ; x), \quad x \in E .
$$

4.2. The large deviations problem for a random evolution process. We consider the problem for the series scheme with a small parameter $\varepsilon \rightarrow 0$ that normalizes the random evolution process $\eta^{\varepsilon}(t ; x), æ^{\varepsilon}(t), t \geq 0$. The problem is stated for the generator

$$
L^{\varepsilon} \varphi(u, x)=\left[Q^{\varepsilon}+\Gamma^{\varepsilon}(x)\right] \varphi(u, x) .
$$

Under the additional conditions introduced in Section 2 when discussing Steps 2-4, the large deviations problem for a random evolution process is reduced to the asymptotic analysis of the normalized exponential generator:

$$
H^{\varepsilon} \varphi^{\varepsilon}(u, x)=e^{-\varphi^{\varepsilon} / \varepsilon} \varepsilon L^{\varepsilon} e^{\varphi^{\varepsilon} / \varepsilon}, \quad \varepsilon \rightarrow 0 .
$$

Below we consider only two possible cases of the large deviations problem for a random evolution process.

4.2.1. The scheme of asymptotically small diffusion [5]-[12, [14]. A random evolution process in the series scheme is determined by its normalized generator

$$
\begin{gathered}
L^{\varepsilon} \varphi(u, x)=\left[\varepsilon^{-3} Q+\Gamma^{\varepsilon}(x)\right] \varphi(u, x), \\
\Gamma^{\varepsilon} \varphi(u)=\varepsilon^{-3} \int_{\mathbf{R}}\left[\varphi\left(u+\varepsilon^{2} v\right)-\varphi(u)\right] \Gamma(d v ; x), \quad x \in E .
\end{gathered}
$$

If the balance condition $\int_{\mathbf{R}} v \Gamma(d v ; x) \equiv 0, x \in E$, holds, then one obtains the asymptotic representation

$$
\begin{gathered}
\Gamma^{\varepsilon} \varphi(u)=\varepsilon B(x) \varphi(u)+\varepsilon R_{\varepsilon} \varphi(u), \quad R_{\varepsilon} \rightarrow 0, \quad \varepsilon \rightarrow 0, \\
B(x) \varphi(u):=\frac{\sigma^{2}(x)}{2} \varphi^{\prime \prime}(u), \quad \sigma^{2}(x):=\int_{\mathbf{R}} v^{2} \Gamma(d v ; x) .
\end{gathered}
$$

Thus, we face the problem of singular perturbation for the operator

$$
L_{0}^{\varepsilon} \varphi(u, x)=\left[\varepsilon^{-3} Q+\varepsilon B(x)\right] \varphi(u, x)
$$


and the main question is on how to reduce the linear problem of singular perturbation (7) to the problem of the asymptotic analysis of the nonlinear exponential generator (5)? The solution of the singular perturbation problem for the operator

$$
H_{0}^{\varepsilon} \varphi^{\varepsilon}(u, x)=e^{-\varphi^{\varepsilon}(u) / \varepsilon} \varepsilon L_{0}^{\varepsilon} e^{\varphi^{\varepsilon}(u) / \varepsilon}
$$

is realized at perturbed test functions

$$
\varphi^{\varepsilon}(u, x)=\varphi(u)+\varepsilon \ln \left[1+\varepsilon \varphi_{1}(u, x)\right] .
$$

The following asymptotic representation

$$
\begin{gathered}
H_{0}^{\varepsilon} \varphi^{\varepsilon}(u, x)=Q \varphi_{1}(u, x)+\widetilde{B}(x) \varphi(u)+R_{\varepsilon}(x) \varphi(u), \quad R_{\varepsilon} \rightarrow 0, \quad \varepsilon \rightarrow 0, \\
\widetilde{B}(x) \varphi(u):=\frac{1}{2} \sigma^{2}(x)\left[\varphi^{\prime}(u)\right]^{2}, \quad \sigma^{2}(x):=\int_{\mathbf{R}} v^{2} \Gamma(d v, x)
\end{gathered}
$$

is the main result (see [5]).

The solution of the problem of singular perturbation for (8) determines the limit exponential generator

$$
\widehat{H} \varphi(u)=\frac{1}{2} \widehat{\sigma}^{2}\left[\varphi^{\prime}(u)\right]^{2}, \quad \widehat{\sigma}^{2}:=\int_{E} \pi(d x) \sigma^{2}(x) .
$$

4.2.2. The scheme of the Poisson approximation [13]-17, [20]. The approach used to solve the large deviations problem in the scheme of the Poisson approximation as well as in the scheme of split and double merging differs from the classical approach (see [3, 4, and from the methods proposed in [5]-[14]. Some further details can be found in [15]- 20].

The large deviations problem in the series scheme is determined by the normalized generator:

$$
\begin{gathered}
L_{\delta}^{\varepsilon} \varphi(u, x)=\left[\varepsilon^{-3} Q+\Gamma_{\delta}^{\varepsilon}(x)\right] \varphi(u, x), \\
\Gamma_{\delta}^{\varepsilon}(x) \varphi(u)=\varepsilon^{-2} \int_{\mathbf{R}}[\varphi(u+\varepsilon v)-\varphi(u)] \Gamma_{\delta}(d v ; x), \quad x \in E .
\end{gathered}
$$

The conditions for the Poisson approximation are as follows:

$\mathrm{P} 1: b_{\delta}(x):=\int_{\mathbf{R}} v \Gamma_{\delta}(d v ; x)=\delta\left[b(x)+\theta_{b}(x)\right]$,

$\mathrm{P} 2: c_{\delta}(x):=\int_{\mathbf{R}} v^{2} \Gamma_{\delta}(d v ; x)=\delta\left[c(x)+\theta_{c}(x)\right]$,

P3: $\Gamma_{g}^{\delta}(x):=\int_{\mathbf{R}} g(v) \Gamma_{\delta}(d v ; x)=\delta\left[\Gamma_{g}(x)+\theta_{\Gamma}(x)\right]$.

The problem of singular perturbation for the nonlinear operator

$$
H_{\Gamma}^{\varepsilon, \delta} \varphi_{\delta}^{\varepsilon}(u, x)=e^{-\varphi_{\delta}^{\varepsilon}(u) / \epsilon} \varepsilon L_{\delta}^{\varepsilon} e^{\varphi_{\delta}^{\varepsilon}(u) / \varepsilon}, \quad \varepsilon, \delta \rightarrow 0, \quad \varepsilon^{-1} \delta \rightarrow 1,
$$

is considered at perturbed test functions

$$
\varphi_{\delta}^{\varepsilon}(u, x)=\varphi(u)+\varepsilon \ln \left[1+\delta \varphi_{1}(u, x)\right] .
$$

The following asymptotic representation is the main result of [16]:

$$
H_{\Gamma}^{\varepsilon, \delta} \varphi_{\delta}^{\varepsilon}(u, x)=Q \varphi_{1}(u, x)+H_{\Gamma}(x) \varphi(u)+\theta_{\Gamma}^{\varepsilon, \delta}(x) \varphi(u),
$$

where

$$
H_{\Gamma}(x) \varphi(u)=b(x) \varphi^{\prime}(u)+\int_{\mathbf{R}}\left[e^{v \varphi^{\prime}(u)}-1\right] \Gamma_{0}(d v ; x), \quad x \in E,
$$

(cf. Section 3.3). 
The solution of the problem of singular perturbation for (9)-(10) determines the limit exponential generator

$$
\begin{gathered}
\widehat{H} \varphi(u)=\widehat{b} \varphi^{\prime}(u)+\int_{\mathbf{R}}\left[e^{v \varphi^{\prime}(u)}-1\right] \widehat{\Gamma}_{0}(d v), \\
\widehat{b}:=\int_{E} \pi(d x) b(x), \quad \widehat{\Gamma}_{0}(d v):=\int_{E} \pi(d x) \Gamma_{0}(d v ; x) .
\end{gathered}
$$

The limit exponential generator (11) contains the jump part defined by the measure of intensity of jumps $\widehat{\Gamma}_{0}(d v)$.

\section{LARGE DEVIATIONS PROBLEM IN THE SCHEME OF SPLiT AND DOUble MERGING}

Consider a switching Markov process $x^{\varepsilon}(t), t \geq 0$, defined on a standard phase space $(E, \mathcal{E})$ in the series scheme with a small parameter $\varepsilon \rightarrow 0, \varepsilon>0$, where

$$
E=\bigcup_{k=1}^{N} E_{k}, \quad E_{k} \cap E_{k^{\prime}}=\varnothing, k \neq k^{\prime} .
$$

Here, the Markov kernel is given by

$$
Q^{\varepsilon}(x, B, t)=P^{\varepsilon}(x, B)\left[1-e^{-q(x) t}\right], \quad x \in E, B \in \mathcal{E}, t \geq 0 .
$$

The operator $Q^{\varepsilon}$ admits the following representation

$$
Q^{\varepsilon}=Q+\varepsilon Q_{1}, \quad Q_{1}(x)=q(x) \int_{E} P_{1}(x, d y) \varphi(y) .
$$

5.1. Large deviations problem under the condition of local balance $\Lambda \mathrm{B}([14])$. The random evolution process is studied under the condition

$\Lambda \mathrm{B}: b(u ; x):=\int_{\mathbf{R}} v \Gamma(u, d v ; x) \equiv 0$

with the following normalization

$$
\xi^{\varepsilon}(t)=\varepsilon^{2} \xi\left(t / \varepsilon^{3}\right), \quad x_{t}^{\varepsilon}:=æ^{\varepsilon}\left(t / \varepsilon^{3}\right) .
$$

The corresponding generator is of the form

$$
\begin{gathered}
\mathbf{L}_{\Lambda}^{\varepsilon} \varphi(u, x)=\left[\varepsilon^{-3} Q+\varepsilon^{-2} Q_{1}+\Gamma^{\varepsilon}(x)\right] \varphi(u, x), \\
\Gamma^{\varepsilon}(x) \varphi(u)=\varepsilon^{-3} \int_{\mathbf{R}}\left[\varphi\left(u+\varepsilon^{2} v\right)-\varphi(u)\right] \Gamma(u, d v ; x) .
\end{gathered}
$$

The generator admits the following asymptotic representation

$$
\mathbf{L}_{\Lambda}^{\varepsilon} \varphi(u, x)=\left[\varepsilon^{-3} Q+\varepsilon^{-2} Q_{1}+\varepsilon \mathbf{B}(x)\right] \varphi(u, x)+\varepsilon \delta^{\varepsilon}(u, x) \varphi(u, x) .
$$

Here,

$$
\mathbf{B}(x) \varphi(u)=\frac{1}{2} B(u ; x) \varphi^{\prime \prime}(u), \quad B(u ; x)=\int_{\mathbf{R}} v^{2} \Gamma(u, d v ; x) .
$$

The exponential generator of the random evolution process is determined by the relation

$$
\begin{gathered}
\mathbf{H} \varphi(u)=\frac{1}{2} \widehat{\widehat{B}}(u)\left[\varphi^{\prime}(u)\right]^{2}, \\
\widehat{\widehat{B}}(u)=\sum_{k=1}^{N} \widehat{\pi}_{k} \int_{E_{k}} \pi_{k}(d x) B(u ; x), \quad B(u ; x)=\int_{\mathbf{R}} v^{2} \Gamma(u, d v ; x),
\end{gathered}
$$

provided condition $\Lambda \mathrm{B}$ holds. 
5.2. Large deviations problem under the condition of total balance TB ([14]). Let the condition of total balance hold, namely

TB: for $1 \leq k \leq N$,

$$
b(u ; x)=\int_{\mathbf{R}} v \Gamma(u, d v ; x) \not \equiv 0, \quad \sum_{k=1}^{N} \widehat{\pi}_{k} \widehat{b}_{k}(u)=0, \quad \widehat{b}_{k}(u)=\int_{E_{k}} \pi_{k}(d x) b(u ; x) .
$$

We introduce the following normalization for the random evolution process:

$$
\xi^{\varepsilon}(t)=\varepsilon^{2} \xi\left(t / \varepsilon^{3}\right), \quad \mathfrak{x}_{t}^{\varepsilon}:=\mathfrak{x}^{\varepsilon}\left(t / \varepsilon^{4}\right) .
$$

The corresponding generator is of the form

$$
\mathbf{L}_{T}^{\varepsilon} \varphi(u, x)=\left[\varepsilon^{-4} Q+\varepsilon^{-3} Q_{1}+\Gamma^{\varepsilon}(x)\right] \varphi(u, x),
$$

where

$$
\Gamma^{\varepsilon}(x) \varphi(u)=\varepsilon^{-3} \int_{\mathbf{R}}\left[\varphi\left(u+\varepsilon^{2} v\right)-\varphi(u)\right] \Gamma(u, d v ; x) .
$$

The generator admits the following asymptotic representation

$$
\mathbf{L}_{T}^{\varepsilon} \varphi(u, x)=\left[\varepsilon^{-4} Q+\varepsilon^{-3} Q_{1}+\varepsilon^{-1} \Gamma(x)+\varepsilon \mathbf{B}(x)\right] \varphi(u, x)+\varepsilon \delta^{\varepsilon}(u, x) \varphi(u, x) .
$$

Here,

$$
\Gamma(x) \varphi(u):=b(u ; x) \varphi^{\prime}(u) .
$$

If condition TB holds, then the exponential generator of the random evolution process is determined by the following relation:

$$
\mathbf{H} \varphi(u)=\frac{1}{2} \widehat{\widehat{B}}_{T}(u)\left[\varphi^{\prime}(u)\right]^{2}, \quad \widehat{\widehat{B}}_{T}(u)=\widehat{\widehat{B}}(u)+\widehat{\widehat{B}}_{0}(u) .
$$

Here,

$$
\begin{gathered}
\widehat{\widehat{B}}(u):=\sum_{k=1}^{N} \widehat{\pi}_{k} \int_{E_{k}} \pi_{k}(d x) B(u ; x), \quad B(u ; x)=\int_{\mathbf{R}} v^{2} \Gamma(u, d v ; x), \\
\widehat{\widehat{B}}_{0}(u):=\widehat{\Pi} \widehat{b}(u, \widehat{x}) \widehat{R} \widehat{\underline{b}}(u, \widehat{x}) \widehat{\Pi}=\sum_{k, l=1}^{N} \widehat{\pi}_{k} \widehat{b}_{k} \widehat{R}_{k l}^{0} \widehat{b}_{l} .
\end{gathered}
$$

\section{Concluding REMARKS}

We have studied the nonlinear exponential generators for the following classes of stochastic processes.

1. Markov random evolutions with locally independent increments in the scheme of a small diffusion.

2. Markov random evolutions with independent increments in the scheme of an asymptotically small diffusion in the phase space with split and double merging.

3. Dynamic random evolutions in the scheme of an asymptotically small diffusion.

4. Random evolutions with independent increments in the scheme of Lévy approximation.

5. Impulse processes in the scheme of Lévy approximation.

6. Random evolutions with independent increments in the scheme of Lévy approximation in a phase space with split and double merging.

7. Impulse processes in the scheme of Lévy approximation in a phase space with split and double merging.

8. Impulse storage processes on a phase space with merging. 


\section{BIBLIOGRAPHY}

1. S. N. Ethier and T. G. Kurtz, Markov Processes: Characterization and Convergence, Wiley, New York, 1985. MR 838085

2. J. Feng, Martingale problems for large deviations of Markov processes, Stoch. Process. Appl. 81 (1999), 165-216. MR1694569

3. J. Feng and T. G. Kurtz, Large Deviation for Stochastic Processes, American Mathematical Society, RI, 2006. MR2260560

4. M. J. Freidlin and A. D. Wentzel, Random Perturbations of Dynamical Systems, SpringerVerlag, New York, 1998. MR 1652127

5. V. S. Korolyuk, Problem of large deviations for Markov random evolutions with independent increments in the scheme of asymptotically small diffusion, Ukraïn. Mat. Zh. 62 (2010), no. 5, 643-650; English transl in Ukrainian Math. J. 62 (2010), no. 5, 739-747. MR2888630

6. V. S. Koroliuk, Markov random evolutions with independent increments in the scheme of asymptotically small diffusion, Dopovidi Nat. Akad. Sci. Ukraine (2010), no. 6, 22-26. (Russian) MR 3112727

7. V. S. Koroliuk, Large deviations problems for Markov random evolution with independent increments in the scheme of asymptotically small diffusion, Comm. Statist. Theory Methods 40(19-20) (2011), 3385-3395. MR2860745

8. V. S. Koroliuk, Random evolutions with locally independent increments on increasing time intervals, Ukr. Mat. Visn. 8 (2011), no. 2, 220-240; English transl. in J. Math. Sci. 179 (2011), no. 2, 273-289. MR2883754

9. V. S. Koroliuk, Large deviations problem for random evolutions, Visnyk Kyiv Taras Shevchenko University (2011), no. 25, 4-6. (Russian)

10. V. S. Koroliuk, Dynamic random evolutions on increasing time intervals, Teor. Imovir. Mat. Stat. 85 (2011), 75-83; English transl. in Theory Probab. Math. Statist. 85 (2012), 83-91. MR 2933705

11. V. S. Koroliuk, R. Manca, and G. D'Amico, Storage impulsive processes in the merging phase space, Ukr. Mat. Visn. 10 (2013), no. 3, 333-342; J. Math. Sci. 196 (2014), no. 5, 644-651. MR 3253989

12. V. S. Koroliuk, R. Manca, and G. D'Amico, Storage impulsive processes on increasing time intervals, Teor. İmovir. Mat. Stat. 89 (2013), 64-74; English transl. in Theory Probab. Math. Statist. 89 (2014), 71-78. MR 3235176

13. V. S. Koroliuk and I. V. Samoilenko, Large deviations for impulse storage processes in the scheme of phase merging, Dopovidi Nat. Akad. Sci. Ukraine (2014), no. 7, 28-35. (Ukrainian)

14. V. S. Koroliuk and I. V. Samoilenko, Large deviations for random evolutions in the scheme of asymptotically small diffusion, Modern Stochastics and Applications, Springer Optimization and Its Applications 90 (2014), 201-217. MR.3236076

15. I. V. Samoilenko, Large deviations for impulsive processes in the scheme of Poisson approximation, Ukraïn. Mat. Zh. 64 (2012), no. 11, 1526-1535. English transl. in Ukrainian Math. J. 64 (2013), no. 11, 1727-1738. MR.3104842

16. I. V. Samoilenko, Large deviations for random evolutions with independent increments in the scheme of Poisson approximation, Teor. İmovir. Mat. Stat. no. 85 (2011), 95-101; English transl. in Theory Probab. Math. Statist. 85 (2012), 107-114. MR2933707

17. I. V. Samoilenko, Large deviations for impulsive processes in the scheme of the Lévy approximation, Teor. Imovir. Mat. Stat. no. 88 (2014), 135-143; English transl. in Theory Probab. Math. Statist. 88 (2015), 151-160. MR3112641

18. I. V. Samoilenko and Yu. V. Shusharin, Large deviations for a random evolution with independent increments in the scheme of the Poisson approximation with split and double merging, Zh. Obchysl. Prykl. Mat. 117 (2014), no. 3, 76-86. (Ukrainian) MR.3353628

19. I. V. Samoilenko, Large deviations for random evolutions with independent increments in the scheme of Lévy approximation with split and double merging, Random Oper. Stoch. Equ. 23 (2015), no. 2, 137-149. MR3353628

20. I. V. Samoilenko, Large deviations for random evolutions with independent increments in a scheme of Lévy approximation, Ukr. Mat. Visn. 12 (2015), no. 1, 67-85; English transl. in J. Math. Sci. 210 (2015), no. 1, 52-66. MR3400243 
Institute of Mathematics, National Academy of Science of Ukraine, Tereshchenkivs'Ka Street, 3, Kyiv 01601, Ukraine

E-mail address: vskorol@yahoo.com

Faculty for Computer Science and Cybernetics, National Taras Shevchenko University, Academician Glushkov Avenue, 4, Kyiv 03127, Ukraine

E-mail address: isamoil@i.ua

Received 29/JUNE/2015

Translated by S. KVASKO 